\title{
Systematic Review of Distal End Radius Fracture Treatment
}

A.S.El Gazzar, H.E.Farag and M.A.Abd El Sadek

Orthopedic Surgery Dept., Faculty of Medicine, Benha Univ., Benha, Egypt

E-Mail:mohammed23@gmail.com

\begin{abstract}
Fractures of the distal radius are extremely common injuries and the outcome differs depending on the type of fracture. Normally stable distal radius fracture is treated non-operatively with a favorable result. In the other hand, unstable fracture easily becomes malunited with inadequate treatment. Those point of the consider might have been to give acceptable a precise written works survey around the present surgical administration about distal span fractures. We searched medline by means of PubMed, SCOPUS, Web about Science, Cochrane focal register from claiming regulated Trials (CENTRAL), Also Google researcher starting with their origin till november 2019. The quest retrieved 2089 exceptional records. We after that retained 57 conceivably qualified records for full-texts screening. Finally, 29 investigations were included. There may be no agreement in regards the suitable medicine for distal span fractures, variables that influence nature from claiming an aggregation Throughout recuperation for example, pain, the rate about recovery, constraint for ADLs, Also possibility difficulties will be All the more discriminating On choosing the medication methodology. Personal satisfaction for life relies ahead individuals' activities, lifestyles What's more preferences, as opposed period. The utilization from claiming inward obsession will be on the rise, yet there bring been no extensive scale randomized regulated trials on analyze VLPS should other medicines. In spite of the fact that there will be exactly proof that results of VLPS would Concerning illustration beneficial to elderly patients Similarly as the individuals to junior patients, there may be no verification that these results defend this more invasive, What's more inclined more expensive, system. By further extensive scale investigations are even now required on affirm our discoveries.
\end{abstract}

Keywords: Distal End, Radius Fracture, Internal plate fixation.

\section{Introduction}

Distal span fractures need aid a standout amongst the The greater part regular happening in the united States, second just on hip fractures done elderly, for an evaluated frequency for 643,000 for every year. This carries an expansive monetary load in the elderly alone, with a evaluated medicare framework consumption of $\$ 385$ will \$535 million dollars yearly. Medicine of distal span fractures verifiably need been predominantly Eventually Tom's perusing modest methods including throwing alternately constrained percutaneous obsession. Taking after the discharge of the volar locking plate in the early 2000s, and promptly reports for triumph with inner fixation, Notoriety need consistently expanded for medication for distal span fractures done more youthful populaces [1].

Various investigations have exhibited great results accompanying inner plate fixation, yet prospective randomized controlled trials would restricted to amount Also contemplate plan. "around those elderly, rates of internal obsession expanded starting with $3 \%$ to 1997 to $16 \%$ for over 2,800 doctor look assignments led from April 1, 2009 to March 31, 2010. Other investigations need exhibited increments of 39\% starting with 1999 with 2007. Provided for those helter skelter rate from claiming distal span fractures in the elderly, and the higher cosset about inner fixation, this need profound financial suggestions. Investigations of medicare uses to medicine of distal span fractures discovered that $\$ 170$ million On medicare trusts were went through clinched alongside 2007 , what added up to $32 \%$ about which were to internal obsession. If doctor inclination proceeds will take after progressive patterns to internal fixation, this intimates vast expands to medicare consumption [2].

Previously, addition, you quit offering on that one must additionally think as of those hidatsa costs, for example, such that passing for productivity, as a result these wounds Normal any rate as 1 alternately a greater amount day away from work worth of effort with see An physician, radiographic/routine follow-up, What's more endorsed days of confined movement in any case for medicine sort. Late american Academy of orthopedic Surgeons (AAOS) rules propose weekly radiographic observation for 3 weeks Emulating diminishment and during discontinuance from claiming immobilization. Rates about give back will worth of effort accompanying distal span fractures have been found on make profoundly variable and the individuals who need helter skelter selfreported pain/disability In benchmark would at hazard to prolonged passing from claiming worth of effort days. 16 Despite the most astounding rate (351. 5 for every $100,000)$ about distal span fractures incurred yearly are in the 75 should 84 quite a while agdistis range, there need aid even now significant rates incurred inside the attempting populace [3].

For 2009, AAOS discharged rules to distal span fractures. However, there at present exist huge geographic varieties On inclination for internal obsession through customary shut medication routines (ranging from $4.6 \%$ on $42.1 \%$ for open decrease internal obsession [ORIF]). Those absence of prospective level i or ii investigations abandons medication choices generally In view of particular survey and clinician experience. Koval Furthermore partners found that hand-fellowship surgeons were essentially less averse to treat for interior obsession over nonfellowship-trained surgeons. Present patterns to ORIF need aid thought with be identified with surgeon's conviction that ORIF Furthermore bolted volar plating are connected with bring down muddling rates, exceptional function, and superior fulfillment over percutaneous or outside fixation; however, these have not been totally substantiated in the written works. It is by acknowledged 
that ORIF gives additional stable obsession and facilitates prior extend about movement yet the clinical essentialness for this need not been turned out [4].

Provided for the shared characteristic of the distal span fracture, What's more astonishing inconsistencies done medication practices, this demonstrates the compelling reason for a finer understanding about present medication methods, outcomes, and the require to All the more prospective randomized regulated trials [5].

Those point of the contemplate might have been to provide a deliberate written works Audit something like the current surgical management from claiming distal span fractures.

\section{Materials and methods}

We performed this systematic review and metaanalysis in accordance to the recommendations of the Preferred Reporting Items for Systematic Reviews and Meta-Analyses (PRISMA) statement and Meta-analysis Of Observational Studies in Epidemiology (MOOSE) statement. PRISMA and MOOSE are reporting checklists for Authors, Editors, and Reviewers of Meta-analyses of interventional and observational studies. According to International committee of medical journal association (ICJME), reviewers must report their findings according to each of the items listed in those checklists [1].

\section{Study Selection and Eligibility Criteria}

- Studies that included adults' patients with fractures of distal end of radius ;

- Studies that assessed the effectiveness and safety of the five common techniques: volar locking plate system (VLPS), non-bridging external fixation (non-BrEF), bridging external fixation (BrEF), percutaneous Kirschner-wire fixation (PKF), and cast immobilization (CI)

- Studies that compared those techniques with each other or with no comparison;

- Studies that reported any of the following outcomes: wrist arc of motion, grip strength, DASH score, Volar tilt and ulnar variance, activities of daily living (ADLs), and complications.

We excluded studies with (1) fewer than 10 patients, (2) no information provided about the number of patients lost to follow-up, (3) complications not reported, (4) studies including a surgical technique that combined the use of an external fixator and plate fixation in the same patient, (5) studies including non-standard procedures such as functional casting or intramedullary wire fixation, or (6) studies of fractures associated with either fractures of the distal ulna (not including isolated fractures of the styloid process), fractures of carpal bones, dislocation of the distal radioulnar joint, fractures with vascular injury, or open fractures.

\section{Data extraction}

Data entry and processing were carried out using a standardized Excel sheet and reviewers extracted the data from the included studies. The extracted data included the following domains: (1) Summary characteristics of the included studies; (2) Baseline characteristics of studied populations; and (3) Study outcomes. All reviewers' independently extracted data from the included articles and any discrepancies were solved by discussion.

\section{Dealing with missing data}

Missing standard deviation (SD) of mean change from baseline was calculated from standard error or $95 \%$ confidence interval (CI) according to Altman [6]

\section{Data synthesis}

Continuous outcomes were pooled as mean difference (MD) or standardized mean difference (SMD) using inverse variance method, and dichotomous outcomes will be pooled as relative risk (RR) using Mantel-Haenszel method. The random-effects method was used under the assumption of existing significant clinical and methodological heterogeneity. We performed all statistical analyses using Review Manager (RevMan) 5.3 or Open Meta-analyst for windows.

\section{Results}

In the present study, we searched Medline via PubMed, SCOPUS, Web of Science, Cochrane Central Register of Controlled Trials (CENTRAL), and Google Scholar from their inception till November 2019. The search retrieved 2089 unique records. We then retained 57 potentially eligible records for full-texts screening. Finally, 29 studies were included Fig (1).

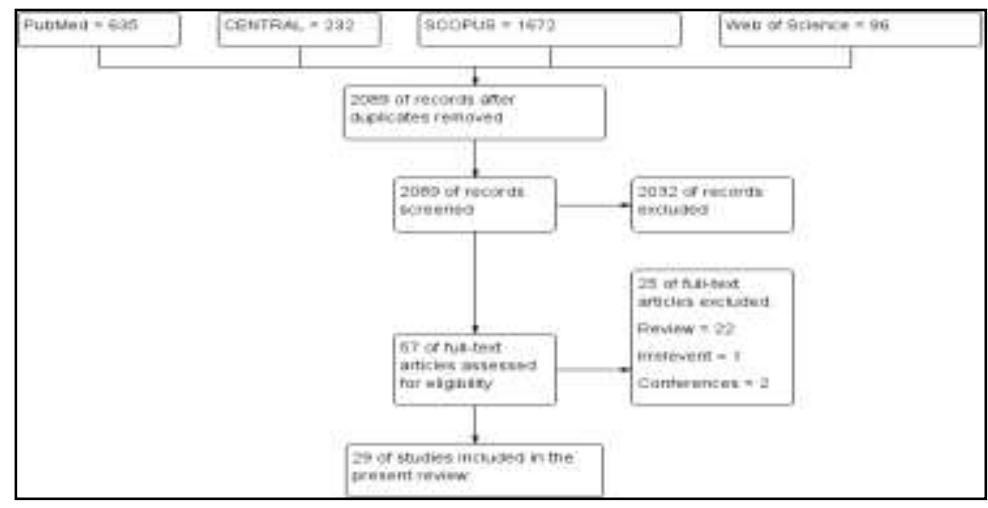

Fig (1) PRISMA flow-chart 
Volar locking plating system, Tables (1-3)

Table (1) Summary Characteristics of the included studies.

\begin{tabular}{|c|c|c|c|c|c|c|c|c|}
\hline $\begin{array}{l}\text { First } \\
\text { Author }\end{array}$ & Year & Procedure & $\mathbf{N}$ & $\begin{array}{l}\text { Study } \\
\text { design }\end{array}$ & $\begin{array}{c}\text { Level of } \\
\text { Evidence }\end{array}$ & $\begin{array}{c}\text { SEQES }^{\wedge} \text { Score } \\
(0-48)\end{array}$ & $\begin{array}{c}\text { Institutional } \\
\text { setting }\end{array}$ & Surgeons \\
\hline Chung & 2008 & VLPS & 25 & PCS & II & 34 & Single site & Single \\
\hline Gerald & 2008 & VLPS & 55 & PCS & II & 24 & Single site & Single \\
\hline Lattmann & 2009 & VLPS & 91 & PCS & II & 34 & Single site & Unknown \\
\hline Arora & 2009 & VLPS, CI & 114 & $\begin{array}{l}\text { Case } \\
\text { series }\end{array}$ & IV & 28 & Single site & Multiple \\
\hline Drobetz & 2003 & VLPS & 50 & $\begin{array}{l}\text { Case } \\
\text { series }\end{array}$ & IV & 13 & Single site & Unknown \\
\hline Orbay & 2004 & VLPS & 24 & $\begin{array}{l}\text { Case } \\
\text { series }\end{array}$ & IV & 21 & Multicenter & Multiple \\
\hline
\end{tabular}

Table (2) Baseline characteristics of the included studies.

\begin{tabular}{|c|c|c|c|c|c|c|c|c|}
\hline $\begin{array}{l}\text { First } \\
\text { Author }\end{array}$ & $\begin{array}{c}\text { No. of } \\
\text { Patients }\end{array}$ & $\begin{array}{c}\text { No. of } \\
\text { Fractures }\end{array}$ & $\begin{array}{c}\text { Female } \\
(\%)\end{array}$ & $\begin{array}{c}\text { Intraarticular } \\
\text { Fracture }(\%)\end{array}$ & $\begin{array}{c}\text { Mean } \\
\text { Age } \\
(y r)\end{array}$ & $\begin{array}{c}\text { Mean } \\
\text { Follow- } \\
\text { Up } \\
(\mathbf{m o})\end{array}$ & $\begin{array}{l}\text { Period of Wrist } \\
\text { Immobilization }\end{array}$ & $\begin{array}{c}\text { Type of } \\
\text { Supplemental } \\
\text { Wrist } \\
\text { Immobilization }\end{array}$ \\
\hline Arora & 53 & 53 & $36(68 \%)$ & $25(47 \%)$ & 76 & 52 & $2 \mathrm{wks}$ & Splint \\
\hline Chung & 25 & 25 & $19(76 \%)$ & $12(48 \%)$ & 69 & 12 & $6 \mathrm{wks}$ & Splint \\
\hline Drobetz & 49 & 50 & $40(82 \%)$ & $34(68 \%)$ & 62 & 26 & $2 \mathrm{wks}^{*}$ & Splint ${ }^{*}$ \\
\hline Gerald & 55 & 55 & $37(67 \%)$ & $55(100 \%)$ & 60 & 29 & 4wks & Cast \\
\hline Lattmann & 91 & 91 & $73(80 \%)$ & $57(63 \%)$ & 64 & 12 & $1-4 \mathrm{wks}$ & Cast \\
\hline Orbay & 23 & 24 & $17(74 \%)$ & $8(33 \%)$ & 79 & 15 & - & - \\
\hline Total & 296 & 298 & $222(75 \%)$ & $191(64 \%)$ & 67 & 25 & - & - \\
\hline
\end{tabular}

Table (3) Final Number of Patients and Fracture, and Result of Scoring System.

\begin{tabular}{lcccc}
\hline Procedure & $\begin{array}{c}\text { First } \\
\text { Author }\end{array}$ & $\begin{array}{c}\text { No. of Patients at } \\
\text { final follow-up }\end{array}$ & $\begin{array}{c}\text { No. of Fractures } \\
\text { at final follow- } \\
\text { up }\end{array}$ & $\begin{array}{c}\text { Scoring system (name: \% of patients } \\
\text { receiving excellent and good } \\
\text { scores or mean points scored) }\end{array}$ \\
\hline VLPS & Arora & 53 & 53 & $\begin{array}{c}\text { Green and O'Brien: } 74 \% ; \text { DASH: } 11.1 / 100^{*} ; \\
\text { PRWE: } 9.3 / 100^{*} \\
\end{array}$ \\
& Chung & 17 & 17 & MHQ: $85 / 100$ \\
Drobetz & 49 & 50 & Cooney: $68 \% ;$ Sarmiento: $92 \%$ \\
Gerald & 55 & 55 & Gartland and Werley: $80 \% ;$ DASH: $7.1 / 100^{*}$ \\
Lattmann & 86 & 86 & DASH: $8.2 / 100^{*}$ \\
Orbay & 23 & 24 &
\end{tabular}

Non-bridging external fixation, Tables (4-6).

Table (2) Summary Characteristics of the included studies.

\begin{tabular}{lcccccccc}
\hline $\begin{array}{l}\text { First } \\
\text { Author }\end{array}$ & Year & Procedure $^{*}$ & $\mathbf{N}$ & $\begin{array}{c}\text { Study } \\
\text { design }\end{array}$ & $\begin{array}{c}\text { Level of } \\
\text { Evidence }\end{array}$ & $\begin{array}{c}\text { SEQES Score } \\
(\mathbf{0}-\mathbf{4 8})\end{array}$ & $\begin{array}{c}\text { Institutional } \\
\text { setting }\end{array}$ & Surgeons \\
\hline Atrosh & 2006 & $\begin{array}{c}\text { Non-BrEF, } \\
\text { BrEF }\end{array}$ & 38 & RCT & I & 38 & Single site & Unknown \\
Krukhaug & 2009 & $\begin{array}{c}\text { Non-BrEF, } \\
\text { BrEF }\end{array}$ & 75 & RCT & I & 41 & Multicenter & Multiple \\
McQueen & 1998 & $\begin{array}{c}\text { Non-BrEF, } \\
\text { BrEF }\end{array}$ & 60 & RCT & I & 39 & Single site & Single \\
\hline
\end{tabular}

Table (3) Baseline characteristics of the included studies. 


\begin{tabular}{lcccccccc}
\hline Procedure & $\begin{array}{c}\text { First } \\
\text { Author }\end{array}$ & $\begin{array}{c}\text { No. of } \\
\text { Patients }\end{array}$ & $\begin{array}{c}\text { No. of } \\
\text { Fractures }\end{array}$ & $\begin{array}{c}\text { Female } \\
(\boldsymbol{\%})\end{array}$ & $\begin{array}{c}\text { Intraarticular } \\
\text { Fracture (\%) }\end{array}$ & $\begin{array}{c}\text { Mean } \\
\text { Age } \\
\text { (yr) }\end{array}$ & $\begin{array}{c}\text { Mean } \\
\text { Follow-Up } \\
(\mathbf{m o})\end{array}$ & $\begin{array}{c}\text { Period of Wrist } \\
\text { Immobilization }\end{array}$ \\
\hline Non-BrEF & Atroshi & 18 & 18 & $16(89 \%)$ & $8(44 \%)$ & 70 & 12 & $6 \mathrm{wks}$ \\
& Krukhaug & 35 & 35 & - & 0 & 62 & 12 & $6 \mathrm{wks}$ \\
& McQueen & 28 & 30 & $27(96 \%)$ & $9(30 \%)$ & 62 & 12 & $6 \mathrm{wks}$ \\
\hline
\end{tabular}

Table (4) Final Number of Patients and Fracture, and Result of Scoring System.

\begin{tabular}{lcccc}
\hline Procedure & First Author & $\begin{array}{c}\text { No. of Patients } \\
\text { at final follow- } \\
\text { up }\end{array}$ & $\begin{array}{c}\text { No. of Fractures } \\
\text { at final follow-up }\end{array}$ & $\begin{array}{c}\text { Scoring system (name: \% of patients } \\
\text { receiving excellent and good } \\
\text { scores or mean points scored) }\end{array}$ \\
\hline Non-BrEF & Atrosh & 18 & 18 & DASH: $11 / 100^{*} ;$ SF-12: $49 / 100$ \\
& Krukhaug & 35 & 35 & DASH: $9 / 100^{*}$ \\
& McQueen & 28 & 28 & \\
\hline
\end{tabular}

Bridging external fixation, Tables (7-9).

Table (5) Summary Characteristics of the included studies.

\begin{tabular}{lcccccccc}
\hline $\begin{array}{l}\text { First } \\
\text { Author }\end{array}$ & Year & Procedure $^{*}$ & N & $\begin{array}{c}\text { Study } \\
\text { design }\end{array}$ & $\begin{array}{c}\text { Level of } \\
\text { Evidence }\end{array}$ & $\begin{array}{c}\text { SEQES }^{\wedge} \text { Score } \\
(\mathbf{0}-\mathbf{4 8})\end{array}$ & $\begin{array}{c}\text { Institutional } \\
\text { setting }\end{array}$ & Surgeons \\
\hline $\begin{array}{l}\text { McQueen } \\
\text { Schmalholz }\end{array}$ & 1996 & BrEF, CI & 90 & RCT & I & 37 & Single site & Multiple \\
$\begin{array}{l}\text { Pritchett } \\
\text { Cannegieter }\end{array}$ & 1995 & BrEF & 25 & RCT & I & 30 & Single site & Single \\
Hegeman & 1997 & BrEF & 32 & $\begin{array}{c}\text { Case } \\
\text { series }\end{array}$ & IV & 15 & Single site & Single \\
Ziran & 2005 & BrEF & 16 & $\begin{array}{c}\text { Case } \\
\text { series }\end{array}$ & IV & 17 & Single site & Unknown \\
& 2000 & BrEF & 10 & $\begin{array}{c}\text { Case } \\
\text { series }\end{array}$ & IV & 14 & Single site & Single \\
\hline
\end{tabular}

Table (6) Baseline characteristics of the included studies.

\begin{tabular}{|c|c|c|c|c|c|c|c|c|}
\hline Procedure & $\begin{array}{c}\text { First } \\
\text { Author }\end{array}$ & $\begin{array}{c}\text { No. of } \\
\text { Patients }\end{array}$ & $\begin{array}{c}\text { No. of } \\
\text { Fractures }\end{array}$ & $\begin{array}{c}\text { Female } \\
(\%)\end{array}$ & $\begin{array}{l}\text { Intraarticular } \\
\text { Fracture }(\%)\end{array}$ & $\begin{array}{c}\text { Mean } \\
\text { Age } \\
(y r) \\
\end{array}$ & $\begin{array}{c}\text { Mean } \\
\text { Follow- } \\
\text { Up (mo) }\end{array}$ & $\begin{array}{l}\text { Period of Wrist } \\
\text { Immobilization }\end{array}$ \\
\hline \multirow[t]{10}{*}{$\mathrm{BrEF}$} & Atroshi & 18 & 19 & $15(83 \%)$ & $11(58 \%)$ & 71 & 12 & $6 w k s$ \\
\hline & Cannegieter & 32 & 32 & $27(84 \%)$ & $19(59 \%)$ & 68 & 36 & $5 w k s$ \\
\hline & Hegeman & 16 & 16 & $16(100 \%)$ & $16(100 \%)$ & 67 & 48 & $6 w k s$ \\
\hline & Krukhaug & 37 & 37 & - & 0 & 62 & 12 & $6 w k s$ \\
\hline & McQueen & 28 & 30 & $28(100 \%)$ & $5(17 \%)$ & 61 & 12 & $6 w k s$ \\
\hline & McQueen & 28 & 30 & $25(89 \%)$ & $16(53 \%)$ & 63 & 12 & $5 w k s$ \\
\hline & Pritchett & 50 & 50 & $26(52 \%)$ & $50(100 \%)$ & 65 & 24 & $6 w k s$ \\
\hline & Schmalholz & 25 & 25 & $24(96 \%)$ & 0 & 66 & 12 & $5 w k s$ \\
\hline & Ziran & 10 & 10 & - & $10(100 \%)$ & 62 & 29 & $8 w k s$ \\
\hline & Total & 244 & 249 & - & $127(51 \%)$ & 65 & 20 & - \\
\hline
\end{tabular}

Table (7) Final Number of Patients and Fracture, and Result of Scoring System.

\begin{tabular}{lcccc}
\hline Procedure & First Author & $\begin{array}{c}\text { No. of Patients at } \\
\text { final follow-up }\end{array}$ & $\begin{array}{c}\text { No. of Fractures at } \\
\text { final follow-up }\end{array}$ & $\begin{array}{c}\text { Scoring system (name: \% of patients } \\
\text { receiving excellent and good } \\
\text { scores or mean points scored) }\end{array}$ \\
\hline BrEF & Atroshi & 18 & 18 & DASH: $7 / 100^{*} ;$ SF-12: $48 / 100$ \\
& Cannegieter & 32 & 32 & Sarmiento: $100 \%$ \\
& Hegeman & 16 & 16 & Gartland and Werley: $63 \%$ \\
& Krukhaug & 37 & 37 & DASH: $13 / 100^{*}$ \\
& McQueen & 28 & 28 & \\
& McQueen & 28 & 28 &
\end{tabular}

Table (9) Continue 


\begin{tabular}{cccc}
\hline Pritchett & 50 & 50 & Lindstrom: $84 \%$ \\
Schmalholz & 25 & 25 & Lindstrom: $84 \%$ \\
Ziran & 10 & 10 & PRWE: pain - $11 / 100^{*}$, disability $-9.8 / 100^{*}$ \\
\hline
\end{tabular}

Percutaneous Kirschner-wire fixation, Tables (10-12).

Table (8) Summary Characteristics of the included studies.

\begin{tabular}{lcccccccc}
\hline First Author & Year & Procedure $^{*}$ & $\mathbf{N}$ & $\begin{array}{c}\text { Study } \\
\text { design }\end{array}$ & $\begin{array}{c}\text { Level of } \\
\text { Evidence }\end{array}$ & $\begin{array}{c}\text { SEQES } \\
(\mathbf{0}-\mathbf{4 8})\end{array}$ & $\begin{array}{c}\text { Score } \\
\text { setting }\end{array}$ & $\begin{array}{c}\text { Surgeons } \\
\text { Fujii }\end{array}$ \\
Hede & 2002 & PKF & 22 & Case series & IV & 17 & Single site & Unknown \\
Shiota & 2000 & PKF & 42 & Case series & IV & 17 & Single site & Unknown \\
van Aaken & 2003 & PKF & 37 & Case series & IV & 18 & Single site & Unknown \\
\hline
\end{tabular}

Table (9) Baseline characteristics of the included studies.

\begin{tabular}{|c|c|c|c|c|c|c|c|c|c|}
\hline Procedure & $\begin{array}{c}\text { First } \\
\text { Author }\end{array}$ & $\begin{array}{c}\text { No. of } \\
\text { Patients }\end{array}$ & $\begin{array}{c}\text { No. of } \\
\text { Fractures }\end{array}$ & $\begin{array}{c}\text { Female } \\
(\%)\end{array}$ & $\begin{array}{c}\text { Intraarticular } \\
\text { Fracture }(\%)\end{array}$ & $\begin{array}{c}\text { Mean } \\
\text { Age } \\
(\mathbf{y r})\end{array}$ & $\begin{array}{c}\text { Mean } \\
\text { Follow- } \\
\text { Up } \\
(\mathbf{m o}) \\
\end{array}$ & $\begin{array}{l}\text { Period of Wrist } \\
\text { Immobilization }\end{array}$ & $\begin{array}{c}\text { Type of } \\
\text { Supplemental } \\
\text { Wrist } \\
\text { Immobilization } \\
\end{array}$ \\
\hline \multirow[t]{6}{*}{ PKF } & Azzopardi & 27 & 27 & $23(85 \%)$ & 0 & 72 & 12 & unknown & Cast \\
\hline & Fujii & 22 & 22 & $21(95 \%)$ & $14(64 \%)$ & 69 & 24 & $5 w k s$ & Unknown \\
\hline & Hede & 42 & 43 & $31(74 \%)$ & 0 & 61 & 33 & $5 \mathrm{wks}$ & Splint \\
\hline & van Aaken & 34 & 34 & $26(76 \%)$ & $19(56 \%)$ & 63 & 30 & 6wks & Splint \\
\hline & Shiota & 37 & 37 & $11(30 \%)$ & $15(41 \%)$ & 66 & 28 & $3 w k s$ & Splint \\
\hline & Total & 162 & 163 & $112(69 \%)$ & $48(29 \%)$ & 66 & 25 & - & - \\
\hline
\end{tabular}

Table (10) Final Number of Patients and Fracture, and Result of Scoring System.

\begin{tabular}{ccccc}
\hline Procedure & First Author & $\begin{array}{c}\text { No. of Patients } \\
\text { at final follow- } \\
\text { up }\end{array}$ & $\begin{array}{c}\text { No. of Fractures } \\
\text { at final follow-up }\end{array}$ & $\begin{array}{c}\text { Scoring system (name: \% of patients } \\
\text { receiving excellent and good } \\
\text { scores or mean points scored) }\end{array}$ \\
\hline PKF & 27 & 27 & $\begin{array}{c}\text { Sheehan's ADL: unilateral }-7.6 / 8, \\
\text { bilateral - } 9.7 / 12\end{array}$ \\
& Azzopardi & 22 & 22 & Saito: $91 \%$ \\
& Fujii & 42 & 43 & Luca: $93 \%$ \\
Hede & 37 & 37 & Gartland and Werley: $85 \%$ \\
Shiota & 25 & 25 & \\
\hline
\end{tabular}

Cast immobilization, Table (13-15)

Table (11) Summary Characteristics of the included studies.

\begin{tabular}{lcccccccc}
\hline $\begin{array}{l}\text { First } \\
\text { Author }\end{array}$ & Year & Procedure $^{*}$ & N & $\begin{array}{c}\text { Study } \\
\text { design }\end{array}$ & $\begin{array}{c}\text { Level of } \\
\text { Evidence }\end{array}$ & $\begin{array}{c}\text { SEQES }^{\wedge} \text { Score } \\
(\mathbf{0}-\mathbf{4 8})\end{array}$ & $\begin{array}{c}\text { Institutional } \\
\text { setting }\end{array}$ & Surgeons \\
\hline $\begin{array}{l}\text { Sanchez- } \\
\text { Sotelo }\end{array}$ & 2000 & CI & 55 & RCT & I & 31 & Single site & Single \\
Young & 2003 & CI & 25 & Caseseries & IV & 18 & Multicenter & Multiple \\
\hline
\end{tabular}

Table (12) Baseline characteristics of the included studies.

\begin{tabular}{lcccccccc}
\hline Procedure & $\begin{array}{c}\text { First } \\
\text { Author }\end{array}$ & $\begin{array}{c}\text { No. of } \\
\text { Patients }\end{array}$ & $\begin{array}{c}\text { No. of } \\
\text { Fractures }\end{array}$ & $\begin{array}{c}\text { Female } \\
(\boldsymbol{\%})\end{array}$ & $\begin{array}{c}\text { Intraarticular } \\
\text { Fracture (\%) }\end{array}$ & $\begin{array}{c}\text { Mean } \\
\text { Age } \\
(\mathbf{y r})\end{array}$ & $\begin{array}{c}\text { Mean } \\
\text { Follow- } \\
\text { Up (mo) }\end{array}$ & $\begin{array}{c}\text { Period of Wrist } \\
\text { Immobilization }\end{array}$ \\
\hline CI & Arora & 61 & 61 & $42(69 \%)$ & $30(49 \%)$ & 81 & 62 & $6 \mathrm{wk}$ \\
& Azzopardi & 27 & 27 & $25(93 \%)$ & 0 & 71 & 12 & $5 \mathrm{wk}$ \\
& McQueen & 30 & 30 & $28(93 \%)$ & $19(63 \%)$ & 64 & 12 & $6 \mathrm{wk}$ \\
& Sanchez- & 55 & 55 & $49(89 \%)$ & $20(36 \%)$ & 67 & 12 & $6 \mathrm{wk}$ \\
Sotelo & & & & & & & & \\
Table (14) Continue & & & & & & & \\
\end{tabular}




\begin{tabular}{cccccccc}
\hline Young & 66 & 66 & $58(89 \%)$ & $9(14 \%)$ & 60 & 84 & $6 \mathrm{wk}$ \\
Total & 239 & 239 & $203(85 \%)$ & $78(33 \%)$ & 69 & 45 & - \\
\hline
\end{tabular}

Table (13) Final Number of Patients and Fracture, and Result of Scoring System.

\begin{tabular}{ccccc}
\hline Procedure & First Author & $\begin{array}{c}\text { No. of Patients } \\
\text { at final follow- } \\
\text { up }\end{array}$ & $\begin{array}{c}\text { No. of } \\
\text { Fractures at } \\
\text { final follow-up }\end{array}$ & $\begin{array}{c}\text { Scoring system (name: \% of patients } \\
\text { receiving excellent and good } \\
\text { scores or mean points scored) }\end{array}$ \\
\hline CI & 61 & 61 & $\begin{array}{c}\text { Green and O'Brien: } 85 \% \text {; DASH: } \\
11.6 / 100^{*} ; \text { PRWE: } 16.9 / 100^{*}\end{array}$ \\
& Arora & 27 & 27 & Sheehan's ADL: unilateral - 7.4/8, bilateral \\
& Azzopardi & 28 & 28 & $-9.4 / 12$ \\
& $\begin{array}{c}\text { McQueen } \\
\text { Sanchez- } \\
\text { Sotelo }\end{array}$ & 55 & 55 & Cooney: $55 \%$ \\
Young & 49 & 49 & Gartland and Werley: $96 \%$ \\
\hline
\end{tabular}

Closed Reduction, Tables (16-17).

Table (14) Summary Characteristics of the included studies.

\begin{tabular}{lccccc}
\hline & Country & n (FTT) & $\begin{array}{c}\text { Median age } \\
\text { (range) }\end{array}$ & $\begin{array}{c}\text { Sex (female } \\
\text { \%) }\end{array}$ & Inclusion period \\
\hline Earnshaw et al & UK & $223(112)$ & $65(15-92)$ & 77 & Aug 1997-Oct 1998 \\
Holkenborg et al & Netherlands & $144(66)$ & $66(\mathrm{~N} / \mathrm{A})$ & 92 & Jun 2008-Jul 2011 \\
Kongsholm et al & Sweden & $116(62)$ & $62(19-86)$ & 91 & N/A \\
\hline
\end{tabular}

Table (15) Study results.

\begin{tabular}{|c|c|c|c|c|c|c|c|}
\hline & & \multicolumn{2}{|c|}{ Presentation } & \multicolumn{2}{|c|}{ Post-reduction } & \multirow[t]{2}{*}{ Pain } & \multirow{2}{*}{$\begin{array}{c}\text { Success rate } \\
(\%)\end{array}$} \\
\hline & & $\begin{array}{c}\text { Dorsal } \\
\text { tilt (dgr) }\end{array}$ & $\begin{array}{c}\text { Shortening } \\
(\mathbf{m m})\end{array}$ & $\begin{array}{c}\text { Dorsal tilt } \\
\text { (dgr) }\end{array}$ & $\begin{array}{c}\text { Shortening } \\
(\mathbf{m m})\end{array}$ & & \\
\hline \multirow[t]{2}{*}{$\begin{array}{l}\text { Earnshaw et } \\
\text { al }^{14}\end{array}$} & $\begin{array}{l}\text { Finger-trap } \\
\text { traction }\end{array}$ & $\begin{array}{c}23.6+/- \\
12.0\end{array}$ & $5.5+/-3.9$ & $-2.5+/-2.0$ & $1.9+/-1.0$ & - & 87.0 \\
\hline & $\begin{array}{l}\text { Manual } \\
\text { traction }\end{array}$ & $\begin{array}{c}24.4+/- \\
10.8\end{array}$ & $7.0+/-5.5$ & $-3.6+/-2.2$ & $2.0+/-1.0$ & - & 87.0 \\
\hline \multirow[t]{2}{*}{$\begin{array}{l}\text { Holkenborg et } \\
\text { al }^{7}\end{array}$} & $\begin{array}{l}\text { Finger-trap } \\
\text { traction }\end{array}$ & $\begin{array}{c}27.4+/- \\
12.0\end{array}$ & $3.8+/-3.9$ & $5.3+/-9.3$ & $0.2+/-2.8$ & 44 & 71.2 \\
\hline & $\begin{array}{l}\text { Manual } \\
\text { traction }\end{array}$ & $\begin{array}{c}28.7+/- \\
11.6\end{array}$ & $5.3+/-3.6$ & $2.7+/-9.6$ & $0.8+/-2.9$ & 53 & 80.5 \\
\hline \multirow[t]{2}{*}{$\begin{array}{l}\text { Kongsholm et } \\
\text { al }^{8}\end{array}$} & $\begin{array}{l}\text { Finger-trap } \\
\text { traction }\end{array}$ & $\begin{array}{c}21.8+/- \\
12.8\end{array}$ & $6.6+/-4.4$ & $-0.2+/-4.3$ & $1.3+/-2.5$ & Less & - \\
\hline & $\begin{array}{l}\text { Manual } \\
\text { traction }\end{array}$ & $\begin{array}{c}19.4+/- \\
12.3\end{array}$ & $6.5+/-4.0$ & $-1.9+/-3.8$ & $2.0+/-2.4$ & More & - \\
\hline
\end{tabular}

Functional Outcomes, Tables (18-19).

Table (16) Active Arc of Motion of the Wrist and Forearm at Final Follow-up (Weighted Mean).

\begin{tabular}{lcccccc}
\hline & $\begin{array}{c}\text { VLPS } \\
(\mathbf{n = 2 1 8})\end{array}$ & $\begin{array}{c}\text { Non-BrEF } \\
(\mathbf{n = 1 8})\end{array}$ & $\begin{array}{c}\text { BrEF } \\
(\mathbf{n = 2 8})\end{array}$ & $\begin{array}{c}\text { PKF } \\
(\mathbf{n}=\mathbf{6 8})\end{array}$ & $\begin{array}{c}\text { CI } \\
(\mathbf{n}=137)\end{array}$ & p value \\
\hline $\begin{array}{l}\text { Wrist flexion/extension arc } \\
\text { (degrees) }\end{array}$ & 118 & 118 & 116 & 112 & 130 & $0.68^{\mathrm{a}}$ \\
\begin{tabular}{l} 
Forearm rotation arc (degrees) \\
\hline
\end{tabular} & 168 & 168 & 153 & 140 & 175 & $0.15^{\mathrm{a}}$ \\
\hline
\end{tabular}

Table (17) Grip Strength at Final Follow-up (Weighted Mean) 


\begin{tabular}{lcccccc}
\hline $\begin{array}{l}\text { Grip strength (\% compared with } \\
\text { contralateral ) }\end{array}$ & $\begin{array}{c}\text { VLPS } \\
(\mathbf{n}=235)\end{array}$ & $\begin{array}{c}\text { Non-BrEF } \\
(\mathbf{n}=28)\end{array}$ & $\begin{array}{c}\text { BrEF } \\
(\mathbf{n}=138)\end{array}$ & $\begin{array}{c}\text { PKF } \\
(\mathbf{n}=95)\end{array}$ & $\begin{array}{c}\text { CI } \\
(\mathbf{n}=\mathbf{2 2 0})\end{array}$ & p value \\
\hline Primary literature review & $\mathbf{8 1}$ & 69 & 84 & 74 & 85 & $\mathbf{0 . 7 0 7}$ \\
Secondary literature review & 76 & - & - & 83 & 84 & $<0.001$ \\
\hline
\end{tabular}

Radiographic Outcome, Table (20).

Table (18) Radiographic Parameters at Final Follow-up (Weighted Mean).

\begin{tabular}{|c|c|c|c|c|c|c|c|}
\hline & & VLPS & $\begin{array}{l}\text { Non- } \\
\text { BrEF }\end{array}$ & BrEF & PKF & CI & p value \\
\hline \multirow[t]{2}{*}{$\begin{array}{l}\text { Volar Tilt } \\
\text { (degrees) }\end{array}$} & $\begin{array}{c}\text { Primary literature } \\
\text { review }\end{array}$ & $\begin{array}{c}3.9 \\
(\mathrm{n}=235)\end{array}$ & $\begin{array}{c}6.5 \\
(\mathrm{n}=81)\end{array}$ & $\begin{array}{c}-0.8 \\
(\mathrm{n}=169)\end{array}$ & $3.7(n=52)$ & $-11(n=220)$ & 0.018 \\
\hline & $\begin{array}{c}\text { Secondary } \\
\text { literature review }\end{array}$ & $\begin{array}{c}3.1 \\
(n=94)\end{array}$ & - & $\begin{array}{c}0.3 \\
(\mathrm{n}=35)\end{array}$ & $0.5(n=49)$ & $-11(n=168)$ & $<0.001$ \\
\hline \multirow{2}{*}{$\begin{array}{l}\text { Radial } \\
\text { Inclination } \\
\text { (degrees) }\end{array}$} & $\begin{array}{c}\text { Primary literature } \\
\text { review }\end{array}$ & $\begin{array}{c}13.4 \\
(n=149)\end{array}$ & $\begin{array}{c}13.7 \\
(n=53)\end{array}$ & $\begin{array}{c}13.9 \\
(n=113)\end{array}$ & $21(n=52)$ & $14.8(n=137)$ & 0.182 \\
\hline & $\begin{array}{c}\text { Secondary } \\
\text { literature review }\end{array}$ & $\begin{array}{c}22.8 \\
(n=94)\end{array}$ & - & $\begin{array}{c}19.5 \\
(\mathrm{n}=35)\end{array}$ & $21(n=49)$ & $18.0(n=168)$ & $<0.001$ \\
\hline \multirow{2}{*}{$\begin{array}{l}\text { Ulnar } \\
\text { Variance } \\
(\mathbf{m m})\end{array}$} & $\begin{array}{c}\text { Primary literature } \\
\text { review }\end{array}$ & $\begin{array}{c}1.5 \\
(n=53)\end{array}$ & $\begin{array}{c}1.0 \\
(\mathrm{n}=53)\end{array}$ & $\begin{array}{c}1.1 \\
(\mathrm{n}=81)\end{array}$ & $3.0(n=27)$ & $3.6(n=143)$ & $<0.001$ \\
\hline & $\begin{array}{c}\text { Secondary } \\
\text { literature review }\end{array}$ & $\begin{array}{c}1.5 \\
(\mathrm{n}=53)\end{array}$ & - & $\begin{array}{c}2.4 \\
(\mathrm{n}=35)\end{array}$ & $3.0(n=49)$ & $3.6(n=143)$ & $<0.001$ \\
\hline
\end{tabular}

Complications, Table (21).

Table (19) Summary of Complications.

\begin{tabular}{|c|c|c|c|c|c|c|c|}
\hline & Type & VLPS & Non-BrEF & BrEF & PKF & CI & p value \\
\hline \multirow[t]{3}{*}{ Minor } & Superficial infection & 0 & 25 & 39 & 2 & 0 & \multirow[t]{3}{*}{$<0.001$} \\
\hline & Others & 2 & 0 & 0 & 9 & 0 & \\
\hline & Total $(\%)$ & $2(1 \%)$ & $25(31 \%)$ & $3916 \%)$ & $11(8 \%)$ & 0 & \\
\hline \multirow{5}{*}{$\begin{array}{l}\text { Major not } \\
\text { requiring } \\
\text { Surgery }\end{array}$} & Nerve lesion & 6 & 1 & 10 & 4 & 4 & \multirow[t]{5}{*}{$<0.001$} \\
\hline & CRPS & 9 & 0 & 16 & 2 & 11 & \\
\hline & Early hardware removal & 0 & 0 & 6 & 3 & 0 & \\
\hline & Others & 3 & 0 & 2 & 0 & 0 & \\
\hline & Total $(\%)$ & $18(6 \%)$ & $1(1 \%)$ & $34(14 \%)$ & $9(7 \%)$ & $15(7 \%)$ & \\
\hline \multirow{6}{*}{$\begin{array}{l}\text { Major } \\
\text { requiring } \\
\text { Surgery }\end{array}$} & Tendon rupture/adhesion & 18 & 2 & 0 & 3 & 3 & \multirow[t]{6}{*}{$<0.001$} \\
\hline & Nerve lesion & 2 & 0 & 2 & 0 & 0 & \\
\hline & Infection & 2 & 0 & 1 & 0 & 0 & \\
\hline & $\begin{array}{l}\text { Hardware loosening, } \\
\text { failure or removal }\end{array}$ & 8 & 0 & 0 & 0 & 0 & \\
\hline & Others & 2 & 0 & 2 & 0 & 0 & \\
\hline & Total (\%) & $32(11 \%)$ & $2(3 \%)$ & $5(2 \%)$ & $3(2 \%)$ & $3(1 \%)$ & \\
\hline
\end{tabular}

\section{Discussion}

Distal span fractures (DRFs) might make the practically normal kind for wrist fractures, Furthermore a bimodal conveyance is seen with a crest frequency to persons 18-25 A long time of age Also second top done persons more seasoned over 65 quite some time. Higherenergy damages would additional normal On more youthful patients who need secondary utilitarian requests Also low-energy wounds are All the more normal in the elderly who are at hazard of osteoporosis. Same time DRFs would the second practically normal crack kind in the elderly populace Furthermore An sourball of morbidity/mortality and misfortune from claiming nature about life, those ideal administration will be even now dubious.

An assortment from claiming surgical and nonsurgical choices would accessible for treating DRFs including shut decrease Furthermore plaster casting, Kirschner-wire stabilization, outer fixation, Furthermore open diminishment Also inside obsession (ORIF) with volar bolted plating. Same time there is a pattern towards preservationist administration from claiming DRFs in the elderly and plaster throwing will be common, later Cochrane precise reviews finished up that there might have been insufflate confirmation with focus At with perform surgery, the thing that sort for surgery may be best, Furthermore the thing that nonsurgical medicine will be best to those medication of DRFs. 
Notwithstanding heterogeneity Around studies, outside obsession and Kirschner-wire adjustment show up should a chance to be connected with higher rates about spoiling. Stratifying elderly patients under low-demand What's more high-demand gatherings might move forward the surgical management about DRFs. Previously, inactive patients with low demands, practical results need aid beneficial Regardless of the vicinity of a deformity, while patients for higher requests might profit from crack adjustment with locking volar plates. Volar plating for fixed-angle screws might be that's only the tip of the iceberg suitableness to slow-healing elderly patients who would that's

only the tip of the iceberg powerless with pin-track spoiling Also sooner tendon aggravation prompting break.

Similarly as the ideal oversaw economy of DRFs in the elderly may be uncertain, the reason for this examine might have been to perform a precise Audit and metaanalysis thinking about the conclusions of surgical What's more nonsurgical administration of DRFs over elderly patients.

\section{Conclusion}

There will be no agreement in regards the fitting medication to distal span fractures. Consequently, signs to surgical intercession are judged separately dependent upon the parity about hazard and profit. In there may be no incredible Contrast the middle of practical results What's more ADL you quit offering on that one quite a while then afterward injury, factors that influence personal satisfaction of life Throughout recuperation for example, such that pain, the rate about recovery, constraint from claiming ADLs, Also possibility difficulties will a chance to be All the more incredulous Previously, choosing the medication technique. Nature from claiming term relies once individuals' activities, lifestyles and preferences, as opposed agdistis. The utilization for inside obsession is on the rise, yet there have been no extensive scale randomized controlled trials should analyze VLPS should other medicines. Despite there will be some proof that results for VLPS need aid Similarly as useful clinched alongside elderly patients Likewise the individuals clinched alongside adolescent patients, there is no verification that these conclusions defend this more invasive, Furthermore liable a greater amount expensive, technique. By further vast scale investigations would still necessary should affirm our discoveries.

\section{References}

[1] R. J. Diaz-Garcia, T. Oda, M. J. Shauver, "A systematic review of outcomes and complications of treating unstable distal radius fractures in the elderly," J. Hand Surg. Am, Vol.36( 5) , PP. 824835, 2011.

[2] C. Bartl, D. Stengel, F. Gebhard, "The treatment of displaced Intra-articular distal radius fractures in elderly patients: a Randomized Multi-center Study (ORCHID) of open reduction and volar locking plate fixation versus closed reduction and cast immobilization," Dtsch. Arztebl. Int, Vol.111(46), PP. 779, 2014.

[3] J. Holkenborg, S.-J. Napel, K. Kolkman, "Closed reduction of distal radius fractures: is finger trap traction superior to manual traction?," Ann. Emerg. Med.vol. 62, PP. S66, 2013.

[4] H. Søsborg-Würtz, S. Corap Gellert, J. Ladeby Erichsen, "Closed reduction of distal radius fractures: a systematic review and meta-analysis," EFORT Open Rev, Vol.3(4), PP. 114-120, 2018.

[5] T. W. O'neill et al., "Incidence of distal forearm fracture in British men and women," Osteoporos. Int, Vol.12(7), PP. 555-558, 2001.

[6] D. G. Altmann J. M. Bland, "Standard deviations and standard errors.," BMJ, Vol.331(7521), PP. 903, 2005 . 